\title{
EL TRABAJO SUMERGIDO EN ESPAÑA EN LA PERSPECTIVA DEL ACTA ÚNICA EUROPEA (1993)
}

\author{
Faustino Miguélez Lobo
}

El trabajo sumergido parece ser una característica de los países del sur de Europa - al menos en la importancia que adquiere en el conjunto del mercado de trabajo-, y por tal motivo parece oportuno preguntarse tanto por la incidencia que en este fenómeno tendrá el Acta Única Europea como por las «distorsiones» que aportará al mercado único. Quiero presentar algunas reflexiones al respecto desde la perspectiva de la realidad española del trabajo sumergido. Pero antes conviene sintetizar lo que conocemos respecto al volumen de este fenómeno, así como analizar su evolución en el último período.

\section{EL VOLUMEN DE TRABAJO SUMERGIDO EN ESPAÑA}

En los primeros años 80 se realizan algunos intentos de medición del volumen de renta nacional producida irregularmente, a través de metodologías (Gutman, Tanzi) utilizadas en ottos países; los tesultados son bastante poco convincentes por su llamativa discordancia. Desde ese momento los estudiosos se dedican a analizar los factotes determinantes del fenómeno, las características de la población ocupada irregularmente y a estimar, en estudios sectotiales o territorialmente linitados, el volumen de fuerza de trabajo en dicha situación.

De esta manera se llega a evaluar el volumen de fuerza de trabajo su- 
mergida en áreas en que esta forma de actividad es muy tradicional: calzado y juguetería en Valencia (más del 80 por ciento), textil en Cataluña (entre 25 y 50 por ciento). $O$ bien la incidencia del paro en el trabajo sumergido; hay varios estudios en Cataluña y Andalucía, según los cuales trabaja un $30^{\circ}$ por ciento o más de los parados, que ponen de manifiesto que, en general, se trata de desocupados que ya no cuentan con subsidio de desempleo y están en trabajos sumergidos muy poco consistentes (ocasionales, eventuales, a horas).

En 1985, el Ministerio de Economía y Hacienda lleva a cabo un importante sondeo (a través de una muestra de 65.000 personas) que clasifica los ocupados en regulares e irregulares, según sea cotrecta o no su relación con el sistema de Seguridad Social. Esta encuesta pone de manifiesto que el 22 por ciento del trabajo es irregular, pero que la principal incidencia del mismo se da en la agricultura ( 31 por ciento) y en los servicios ( 23 por ciento) y la menos importante en la industria ( 16 por ciento), que es donde se la había ubicado tradicionalmente; esto pone de manifiesto que hay cambios que convendrá investigar. Dentro de los servicios son los servicios personales, el comercio y hastelería los subsectores en que el trabajo sumergido tiene más importancia; en la industria destacan las industrias manufactureras.

La misma investigación indica otros aspectos que conviene tener presentes, por cuanto pueden señalat diferencias muy importantes con otros países de la CEE: los empresarios - con y sin asalariados-n, tanto agrarios como no agratios, están sobrertepresentados en la actividad sumergida, to mismo que los trabajadores de los servicios; más de un tercio de los sumergidos son no asalariados; el trabajo sumergido es realizado tres veces más por las personas que no son cabezas de familia (el 29 por ciento de este colectivo) que por los que son cabezas de familia (el 10 por ciento), lo que nos permite decir que estos últimos están socialmente trás protegidos por lo que respecta al empleo.

Hay importantes diferencias territoriales dentro de España tanto por lo que se refiere al volumen de trabajo sumergido, como a sus características fundamentales, de manera que las zonas de mayor irregularidad no son precisamente aquellas más industrializadas ni más ricas, sino las más pobres y menos desarrolladas. 


\section{LAS CARACTERISTICAS DEL TRABAJO SUMERGIDO}

Para mejor comprender la naturaleza del trabajo sumergido y su evolución en España en los últimos 10 o 15 años, vamos a diferenciat tres fases:

\section{a) El trabajo sumergido tradicional}

El trabajo sumergido tradicional se ubicaba en zonas de un cierto monocultivo industrial o en la agricultura latifundista. En esta última afectaba a los trabajadores asalariados, en general eventuales, que eran contratados ilegalmente en diversas fases de la producción. En la industria el trabajo sumergido tradicional tenía el apoyo fundamental en el papel social jugado por la familia: la mujer realizaba trabajo no declarado a domicilio para las empresas textiles, de juguetería y otras, al tiempo que realizaba las tareas domésticas: Ios pequeños artesanos o empresarios encontraban gran parte de su fuerza de trabajo sumergida en la familia directa o en los parientes. Igualmente la familia y la mujer constituían el gran volumen de trabajo irregular en los servicios: servicio doméstico no declarado, ayuda familiar no declarada en el comercio y la hostelería.

El bajo coste de esta mano de obta, así como su situación económica y cultural de gran disponibilidad (algunos autores han hablado de mano de obra cautiva) y el escaso control social y estatal (de los sindicatos o de la Administración) sobre las empresas parecen ser los factores determinantes del fenómeno antes de los años setenta. Aparte de este tipo de trabajo sumergido, hay que señalar como algo tradicional la existencia de segundos o terceros empleos irtegulares, en particular en ciertas ocupaciones como empleados públicos, profesionales liberales, trabajadores manuales muy cualificados.

\section{b) La expansión de los años setenta}

Los estudiosos coinciden en señalar que aunque el trabajo sumetgido no nace en España a consecuencia de la crisis económica, sin embargo ésta tiene un papel importante en su desarrollo. Los factores determinantes de ese importante aumento son varios y así se han señalado. Está, en primer lugar, un factor subjetivo-individual: la necesidad de obtener unos ingresos necesarios - a veces no hay otro camino que el sumergido- o de incrementar salarios o pensiones muy bajos o bien escapar al pago de tasas; sin 
embargo, una importante parte de este empleo sumergido recibe ingresos tan bajos que ni siquiera tendría que pagar tasas.

Por otro lado están los factores que subrayan el punto de vista de las empresas; aquí se ha puesto el acento sobre la presunta excesiva rigidez del mercado de trabajo heredado del franquismo, que no permitiría hacer frente a la competencia internacional, refiriéndose tanto al coste de la mano de obra como al presunto excesivo peso de la fiscalidad sobre las empresas. También respecto a este tipo de argumentos cabría objetar que la fiscalidad en España está por debaj̣o de la media europea, al igual que los costes sa. lariales, y que la flexibilidad real del mercado de trabajo - no la teórica, es decir, la que se refleja en convenios y ordenanzas - es más alta de lo que a veces se señala, debido al escaso control que la Administración ha realizado y que los sindicatos han podido realizar sobre el cumplimiento de leyes y normas.

Pero hay un fenómeno que tiene lugar en los años setenta y ptimeros ochenta, a mi entender profundamente explicativo de la expansión del tra* bajo sumergido: se trata de la reestructuración productiva que comporta un enorme proceso de descentralización y acentúa de manera importante la segmentación del mercado de trabajo; dicha segmentación crea muchos puestos de trabajo precarios y legalmente desprotegidos que encuentran una fuerza de trabajo «adecuada» en las mujeres, en los jóvenes que no encuentran empleo, en los trabajadores escasamente cualificados que son expulsados del proceso productivo y en los jubilados, que no pueden vivir de unas pensiones excesivamente bajas. Esto incrementa enormemente el trabajo irregular.

La novedad de estos años es que el trabajo irregular es «organizado», es decir, se extiende a los trabajadotes dependientes en forma de empleos sin contrato, horas extraordinarias no declaradas; en general, las condiciones laborales de estas personas están en la parte baja de la escala: salarios bajos, largas jornadas, poca seguridad e higiene.

Pero las formas tradicionales de trabajo irregular también se acentúan: son muchas más las personas que trabajan a domicilio - ya no sólo mujeres-, crece el autoempleo, en parte legal y en parte sumergido, en sectores como la construcción, el transporte, el comercio, la hostelería. En general, la innovación que aqui se detecta es escasa, aunque hay excepciones como puede ser el sector de la electrónica, ciertos servicios a las empresas, etc. 


\section{c) Los cambios de los años ocbenta}

El cambio fundamental que podemos detectar en los últimos años es lo que podríamos calificar de «legalización de la desregulación», es decir, la aparición de nuevas formas de contratación: contratos en formación, en prácticas, a tiempo parcial y, sobre todo, eventuales. Estas formas de contratación, entre las que predominan los empleos de menos de tres meses de duración, constituyen ahora más del 90 por ciento de los contratos que se realizan a través del INEM, afectando, en particular, a los jóvenes de ambos sexos y a las mujeres.

Estas nuevas formas de contratación no necesariamente eliminan el trabajo estrictamente sumergido. La facilidad con la que se puede contratar y despedir significa que las empresas tienen la posibilidad de teemplazar su antiguo trabajo irregular por trabajo precario. Pero es obvio que estos trabajadores inestables pueden ser contratados por un período breve, trabajar luego sumergidamente durante otro período y volver nuevamente al trabajo regular pero en forma inestable. Eso es lo que sucede con frecuencia según diversos estudiosos del tema.

Se ha creado, así, una franja extraordinariamente flexible de trabajadores -en general jóvenes, mujeres y hombres adultos muy poco cualificados-m que lo mismo pueden estar en situación legal que ilegal, pero que tienen unas condiciones de trabajo idénticas: sus salarios, posibilidades de promoción, seguridad en el empleo, condiciones de salud laboral, jornada, etc., son inestables e inferiores a la que se registran para los trabajadores regulares.

En los últimos años los procesos de reestructuración han incrementado enormemente el autoempleo; es un autoempleo muy móvil, puede ser legal o no, pero en caso de serlo strele tener una orla de trabajo ilegal en su en. torno: familiares, amigos, conocidos trabajando a tiempo parcial. En la misma forma podemos señalar un aumento del segundo trabajo, sobre todo en sectores muy seguros: profesionales, empleados de banca, funcionarios; a menudo este segundo trabajo es irregular, ocupa sólo algunas horas y se tealiza como trabajo independiente.

En este período se han agregado otros factores que determinan tanto el tipo de trabajo sumergido como sus caractetísticas. Las modernas áteas metropolitanas generan un volumen importante de servicios personales y a las empresas que pueden ser cubiertos con empleos de los niveles más bajos del mercado de trabajo y por gente que los acepta a tiempo patcial o por breves períodos. Las posibilidades de que muchos de ellos sean sumergidos se han incrementado.

En ciertos casos el trabajo sumergido es una forma de iniciar, sin tanto 
riesgo, un nuevo negocio, una cooperativa, un instituto de investigación, mientras que la intención es legalizarse luego para darse a conocer; obviamente, esta forma de actuar es posible para quienes tienen un capital o un cierto nivel de formación. Es particularmente en estos casos en los que la innovación va asociada al trabajo irregulat.

\section{ACTA ÚNICA Y TRABAJO SUMERGIDO EN ESPAÑA}

Teniendo en cuenta el panotama descrito, podemos conjeturar qué efectos puede tener el Acta Unica de 1992 sobre el trabajo sumergido tanto en España como para los trabajadores españoles fuera de España.

\section{En España}

a) El trabajo sumergido en España está ligado, en gran parte, a diferencias regionales en la estructura productiva, a tradiciones laborales, al papel de la familia, a la poca capacidad de control del Estado, a la escasa fuerza de los sindicatos. Todo esto no sólo provoca formas de trabajo sumergido diferentes entre Cataluña y Andalucía, por ejemplo, sino también entre Andalucía y la cuenca del Rhur o la Emilia-Romagna. Nada de esto va a cambiar con el Acta Única - aunque algunos de estos factores podrían cambiar a más largo plazo--, por tanto, pocos cambios podemos esperar por este lado.

b) Sin embargo, el Acta Única puede incrementar un proceso que ya está en curso, la descentralización por las empresas de ciertas fases productivas o de ciertos servicios a países en que se dan algunas de las características siguientes: tradición de trabajo sumergido, red muy flexible de pequeña empresa, debilidad institucional o real de los sindicatos, menores controles fiscales, laborales, de seguridad e higiene. En este tipo de países o regiones (España es un caso obvio) y por tales motivos podemos esperar que el trabajo sumergido -en un modelo como el descrito en párrafos anterioresirá en aumento. De hecho, las perspectivas de empleo están despertando en los últimos años en España un colectivo de baja tasa de actividad, las mujeres. Esta disponibilidad facilita el incremento del trabajo sumergido.

c) Al mismo tiempo, los subsectores orientados hacia la exportación y sujetos a competencia internacional fuerte pueden incrementar el uso del trabajo sumergido como vía de reducción de costos. Ese podtía ser el caso de la electrónica, el textil de alta calidad, el calzado y los juguetes. 
d) Profesionales liberales, provenientes de otros países de la Comunidad, que ofrezcan sus servicios a las empresas o a los particulares y mantengan parte de sus actividades irregularmente, pueden instalarse en países de menor control fiscal o profesional, como es el caso de España. También por aquí se dazá un incremento del trabajo irregular, aunque no será cuantitativamente relevante, vía segundo trabajo, por ejemplo.

e) Pot el contrario, la implantación del Acta Única obligará a la ad. ministración española - ya la está obligando- a un mayor rigor fiscal frente a colectivos como los autoempleados y los profesionales liberales, así como frente a las empresas en general. Pero la mejora del control fiscal no necesariamente va a llegar al ámbito del trabajo, puesto que existe una tradicional «complicidad» entre empresarios y trabajadores respecto a este tema, que difícilmente va a ser erradicada en pocos años.

\section{Fuera de España}

a) Se puede pensar que las diferencias salariales y de consumo pueden provocar nuevas migraciones de los países con altas tasas de paro (España en el caso) hacia los países comunitarios con tasas más bajas; ello podría disminuir el trabajo sumergido en los primeros y aumentar la mano de obra disponible para el trabajo irregular en los segundos. A pesar de esa posibilidad teórica, las migraciones no son tan probables, por cuanto hoy el paro masivo es de jóvenes, no de cabezas de familia, y aquéllos tienen una concepción del trabajo, del consumo y del proyecto de vida que los hace poco proclives a emigrar a países distintos para trabajar, lo que no excluye que ése pueda set el caso para un númeto reducido de ellos.

Pero sí es probable otro tipo de migración, la de temporada o por períodos costos, que puede afectar tanto a un tipo de trabajadores adultos y cabezas de familia (los temporeros de la agricultura, principalmente) como a jóvenes sin empleo; los primeros se dirigirán a actividades agrícolas en otros países de la Comunidad y los segundos a actividades de servicios poco cualificadas. Este fenómeno puede incrementar el trabajo sumergido en algunos países de la CEE, por cuanto se tratará de mano de obra de difícil control.

b) En cualquier caso, aun con el tipo de migraciones a las que me he referido, el volumen de trabajo sumergido en España disminuiría poco, al tratarse más bien de personas paradas. 


\section{CONCLUSION}

El Acta Ûnica no influirá, a corto plazo, de manera relevante sobre el trabajo irregular y sus características en los diversos países de la Comunidad, o al menos en aquéllos del sur de Europa como es el caso del nuestro.

A lo sumo se acentuarán las diferencias entre los países del norte y los países del sur (o algunas regiones de los pasíes del sur), incrementándose en estos últimos ciertas formas de trabajo sumergido ya existentes.

Los cambios más en profundidad pueden darse a más latgo plazo, cuando se dé un mayor acercamiento en las estructuras económicas, sociales y culturales de los diversos países. 


\section{REFERENCIAS BIBLIOGRAFICAS}

Angueta, J. y Casas, J. B.: Rubi, un estudi socio-econdmic, Ajuntament de RubíBanca Catalana, 1987.

Aracil, R. y Gatcía Bonafé, M.: «Industria doméstica e industrialización en España», Hacienda Pública Española, 55, 1978.

Annalte, E.: Agricultura a tiempo parcial en el Pais Valenciano, Ministerio de Agricultura, Madrid, 1980.

Balcells, A.: Capitalismo e industrialización en Catalunya, Laia, Barcelona, 1971. Benton, Lauren: «La informalización del trabajo en la industria», Papeles de Economía, núm. 26, Madrid, 1986.

Bernabé, J. M.: «Factores de localización y crisis de la industria valenciana del calzadon, Panorama Bursátil, núra. 6, 1977.

- Economía sumergida en la industria del calzado, Ponencia presentada en el Simposium Internacional sobre economía sumergida, Presidencia del Gobierno, Madrid, 1981.

- «Economía sumergida en la Comunidad Valenciana», Papeles de Economía de las Comunidades Autónomas, Madrid, 1986.

Casals, M. y Vidal Villa, J.M.: L'economia de Sabadell. 2 vols., Ajuntament de Sabadell, 1982.

-m «La industria sumergida: el caso de Sabadell», Papeles de Economía, núm. 22, 1985.

Castillo, J.J. et al.: Difusión industrial y economía informal en la crisis, Madrid, 1984.

Celada, F.: «Transformaciones en el espacio productivo del capital: el sistema de fábrica o fábrica difusa", Sociología del Trabajo, núm. 5, 1981.

CIS y Secretaría General de Economía y Planifcación: Condiciones de vida y trabajo en España, Madrid, 1986.

CCOO: La mujer y la economía sumergida, Secretaría de la mujer, CCOO, Madrid, 1987.

Generaiitat de Catalunya: Enquesta sobre l'atur, Estudis Sectorials, Generalitat de Catalunya, Barcelona, 1984.

Gómez Pérezagua, R.: «Implicaciones micro y macro económicas de la economía 
subterzánea», Información Comercial Española, semanal, Ministerio de Gomercio, Madrid, 1983.

Izquierdo, M. J., Miguélez, F. y Subirats, M.: Enquesta Metropolitana, vol. I, Informe General, Instituto de Estudios Metropolitanos de Barcelona, Barce. lona, 1987.

Jodar, P. y Martín Artiles, A.: Crisis económica y relaciones industriales, Ed. Zero, Madrid, 1984.

Jodar, P. y Lope, A.: Con el agua al cuello, el trabajo en la economía sumergida, Ed. Revolución, Madrid, 1985.

Lafuente, A.: «Comportamiento de la empresa en la economía oculta», ICE, núm. 587, julio, 1982.

López, C.: El textil irregular en Terrassa 1975-85, Ajuntament de Terrassa. Terrâssa, 1986.

Mancha Navarro, T.: «Economía Paralela: un intento de síntesis», ICE, núm. 642, febreto, 1987.

Miguélez, F.: «Economía sumergida y transformaciones socio-laborales», Boletin de Estudios Econbmicos, Deusto, Bilbao, 1982.

- Los sindicatos ante la economia sumergida, Seminario en la Facultad de Económicas de Valencia sobre Economía Sumergida, Valencia, enero 1986.

Miguélez, F. y Recio, A.: «Catalunya: la economia ignota». En: La otra economía, trabajo negro y sector informal. Institut Alfons el Magnánin, Valencia, 1986.

Moltó, M. A.: «La economía irregular, una primera aproximación al caso español», Revista Española de Economia, 1980.

Parra, T.: «La economía subterránea, nueva problemática en la crisis», Pensamiento Iberoamericano, núm. 3, enero, 1983.

Pico, J. y Sanchís, E.: La economía sumergida en España, Seminario de Froscati, Italia, noviembre, 1982.

Recio, A.: Economia stumergida, paro y mercado de trabajo, Generalitat de Catalunya, 1984 (mimeo).

- «Economía sumergida y transformación de las relaciones laborales en España», Papers, Revista de Sociología, núm. 27, Barcelona, 1986.

Recio, A., Miguélez, F. y Alós, R.: La industria textil lanera del Vallés Occidental, CCOO, Barcelona, 1988.

Rodríguez Inciarte, M.: «La economía oculta». Papeles de Economía Española, núm. 10, Madrid, 1982.

Ruesga, S. y De Blas, A.: «Mercado de trabajo y economía oculta en Andalucía», Cuadernos IAR, núm. 3, Sevilla, 1985.

Ruesga Benito, S.: Métodos de estimación de la economía oculta. Su incorporación a los sistemas de cuentas nacionales, Fundación Juan March. Madrid, 1985.

- «Empresa oculta en España», Cinco Dias, DIESA, Madrid, 23 de marzo 1986.

- "¿Emerge la economía oculta?» Boletín del Colegio de Economistas de Madrid, Madrid, 1986. 
Ruesga Benito, S.: «La economía sumergida en España», Revista del Instituto de Estudios Económicos, núm. 1, 1987.

Sáez, F.: «Economía sumergiđa y mercado laboral», IP Intormación de Publicidad y Marketing, núm. 264, Madrid, 1986.

Sanchís, E.: «Industria subterránea y crisis económica en el Paús Valenciano», ICE, núm. 587, 1982.

- «Economía subterránea y descentralización productiva en la industria manufacturera», Boletín de Estudios İconómicos, núm. 117, 1982.

- El trabajo a domicilio en el País Valenciano, Instituto de la Mujer, Madrid, 1984.

- «La economía sumergida: Dr. Jekill o Mr. Hyde», Debats, núm. 10, 1984.

- «Trabajo blanco, negro, malo, bueno», Alfoz, núm. 40, 1987.

Sanchís, E. y Picó, J.: «La economía sumetgida: el estado de la cuestión en España», Sociología del Trabajo, núm. 9, 1983.

Sanchís, E. y Miñana, J. (recopiladores), AA.VV.: La otra economía: trabajo negro y sector informal, Ed. Alfons el Magnànim, Valencia, 1988.

Trigo, J. y Vázquez, C.: La economía irregular, Generalitat de Catalunya, 1983. UGT: La economía sumergida en los sectores textil y piel, Federación Estatal Textil-Piel, UGT, Madrid, 1988.

Ybarta Pérez, J. A.: «Economía subterránea. Reflexiones sobre la crisis económica en España», Economia Industrial, núm. 218, febrero 1982.

- La reconversión oculta en España: principios politicos y consecuencias, Ponencia ALFOZ, Jornadas «El otro empleo», Madrid, 1987. 